Alessandra Fontes Ferreira da SIIVA Guerra

Maria Emília Daudt von der Heyde ${ }^{2}$

RoGÉRIO ANDRADE MULINARI ${ }^{3}$

Artigos originais

Palavras-chaves:

Gravidez na adolescência

Gestação

Peso ao nascer

Recém-nascido

Consumo de alimentos

Estado nutricional

Keywords:

Pregnancy in adolescence

Preganancy

Birth weight

Infant, newborn

Food consumption

Nutritional status

Correspondêncio:

Alessandra Fontes Ferreira da Silva Guerra Rua Victor Konder, 55 - apto. 901 Victor Konder - Blumenau/SC - CEP 89012-170 Fones: (47) $9922-3535$ / (47) 3222-3079

E-mail:alenutry@yahoo.com.br

Recebido

06/01/2006

Aceito com modificacāes $13 / 02 / 2007$

\section{Impacto do estado nutricional no peso ao nascer de recém-nascidos de gestantes adolescentes}

\author{
Nutritional status impact on the birth weight of newborns \\ pregnant adolescents
}

\title{
Resumo
}

OBJETIVO: avaliar o impacto do estado nutricional de gestantes adolescentes no peso do recém-nascido. MÉTODOS: em estudo de coorte histórica foram avaliadas 97 gestantes adolescentes atendidas no período de maio a junho de 2004 e seus recém-nascidos. Foram incluídas gestantes entre 10 e 19 anos, internadas em trabalho de parto, e excluídas aquelas com gravidez múltipla, complicações gestacionais, idade gestacional menor do que 37 semanas e dados incompletos no prontuário. A avaliação do estado nutricional materno incluiu altura, índice de massa corporal (IMC) pré-gestacional, ganho de peso gestacional (GPG) e consumo calórico e protéico, obtidos por relatórios de consumo alimentar habitual no final do terceiro trimestre gestacional. A associação entre as variáveis maternas (altura, IMC pré-gestacional, GPG e consumol e o peso do recém-nascido foi analisada pelo teste de correlação de Spearman. $\bigcirc$ nível de significância de erro $\alpha$ foi de $5 \%(p<0,05)$. RESULTADOS: a média de idade foi de $17,8 \pm 1,12$ anos. A maioria (66\%) iniciou a gestação eutrófica, 29\% com baixo peso e 5\% com sobrepeso. A maioria apresentou GPG e consumo calórico e protéico inadequados. Observou-se que 7\% dos recém-nascidos apresentaram baixo peso e 37\% peso insuficiente. A altura e ○ GPG apresentaram correlação positiva e significante com o peso ao nascer. $\bigcirc$ IMC pré-gestacional e o consumo protéico apresentaram correlação inversa e estatisticamente significante com o peso ao nascer. Não foi observada correlação entre consumo calórico e o peso ao nascer. CONCLUSÃO: A altura materna e - GPG influenciaram o estado nutricional do recém-nascido.

\section{Abstract}

PURPOSE: to evaluate the impact of the nutritional status of pregnant adolescents on the birth weight. METHODS: a cohort study including 97 adolescents and their respective newborns, evaluated from May to June, 2004. Pregnant women from 10 to 19 years old in labor were included in the study, and those with multiple pregnancies, complications, less than 37 weeks gestation, and incomplete data records were excluded. Maternal nutritional status evaluation included height, body mass index (BMI) before pregnancy, gestational weight gain (GWG) and caloric-proteic intake, obtained by habitual food intake recordatory by the end of the third gestational trimester. The association between maternal variables (height, pre-gestational BMI, GWG and intake) and the newborn weight was analyzed by Spearman's correlation test. Statistical significance was assumed when $p<0.05$. RESULTS: the mean age was $17.8 \pm 1.12$ years old. Most adolescents $(66 \%)$ started pregnancy with adequate weight, $29 \%$ had low weight and $5 \%$ overweight. Most adolescents showed inadequate GWG, caloric and proteic intake. Low birth weight was recorded in $7 \%$ of the newborns and insufficient weight was recorded in 37\% of them. Maternal height and GWG showed positive and significant BW relation. Pre-gestational BMI and protein intake showed statistically significant inverse correlation with birth weight. No correlation between caloric intake and BW was demonstrated. CONCLUSION: maternal height and GWG influence the newborn nutritional status.

\section{Hospital e Maternidade Victor Ferreira do Amaral - Curitiba (PR), Brasil.}

'Especialista em Terapia Nutricional com Treinamento em Serviço pela Universidade Federal do Paraná - UFPR - Curitiba (PR), Brasil; Chefe do Serviço de Nutrição do Hospital e Maternidade Victor Ferreira do Amaral - Curitiba (PR), Brasil.

2 Professora Adjunta do Departamento de Nutrição; Vice-Diretora do Setor de Ciências da Saúde Universidade Federal do Paraná - UFPR - Curitiba (PR), Brasil.

${ }_{3}^{3}$ Professor Adjunto de Clínica Médica - Nefrologia; Diretor do Setor de Ciências da Saúde, Universidade Federal do Paraná - UFPR - Curitiba (PR), Brasil. 


\section{Introdução}

A gestação na adolescência tem sido identificada como um problema de saúde pública no Brasil e em outros países, pelo aumento crescente de sua incidência e pelas importantes conseqüências biológicas, sociais e psicológicas, especialmente ao ocorrer de maneira precoce e não planejada ${ }^{1}$.

O prognóstico da gestação é influenciado pelo estado nutricional materno antes e durante a gestação ${ }^{2,3}$, já que boas condições do ambiente uterino favorecerão o desenvolvimento fetal adequado ${ }^{3}$. A inadequação do estado nutricional materno tem grande impacto sobre o crescimento e desenvolvimento do recém-nascido $(\mathrm{RN})$, pois o período gestacional é uma fase em que as necessidades nutricionais estão elevadas, decorrentes dos ajustes fisiológicos da gestante e das demandas de nutrientes para o crescimento fetal ${ }^{4}$.

$\mathrm{Na}$ adolescência, a evolução da gestação e do crescimento fetal é alterada pelos maiores riscos de desnutrição, anemia, deficiências vitamínicas, retardo do crescimento intra-uterino, uso de drogas e infecções, resultando em aumento dos índices de prematuridade, baixo peso ao nascimento e desnutrição pós-natal ${ }^{5,6}$.

A desnutrição é uma causa conhecida de baixo peso ao nascer, especialmente em países em desenvolvimento ${ }^{4}$. Gestantes adolescentes com índice de massa corporal (IMC) de desnutrição no final da gestação têm maior número de RNs com peso menor de 2.500 gramas $^{7}$.

A altura materna, como outras medidas antropométricas, tem sido utilizada para avaliar os riscos de baixo peso ao nascer ${ }^{2,4,8,9}$. Em um estudo realizado em Teresina, no Piauí, a relação da altura materna com as faixas de peso ao nascer evidenciou forte associação estatística, corroborando achados que apontam menor altura com risco de baixo peso ao nascer?

A relação entre ganho de peso da gestante e o peso ao nascer é amplamente conhecida. O IMC, que proporciona informações com relação às reservas energéticas, tem sido utilizado com freqüência para determinar e monitorar o ganho de peso baseado no estado nutricional pré-gestacional ${ }^{3}$.

Gestantes com ganho de peso insuficiente apresentam maiores riscos de gerarem recém-nascidos com peso inadequado, podendo comprometer o crescimento pós-natal, com maior risco de morbidade no primeiro ano de vida ${ }^{2}$.

Outros indicadores do estado nutricional, como o consumo alimentar, também interferem diretamente sobre o resultado do concepto. Quando há consumo inadequado de nutrientes pela mãe, deve-se considerar o período da gestação em que ocorre, o quão grave é essa inadequação e por quanto tempo persiste ${ }^{10}$. Gestantes que apresentam uma reserva inadequada de nutrientes, aliada a uma ingestão dietética insuficiente, poderão ter um comprometimento do crescimento fetal e, conseqüentemente, do peso ao nascer ${ }^{4}$.

O comportamento alimentar da adolescente durante a gestação é influenciado por vários fatores. A preocupação em manter o atual padrão de beleza, muito comum entre as adolescentes, está associada a dietas inadequadas, podendo ocasionar transtornos alimentares, como anorexia e bulimia, comprometendo, assim, o ganho de peso adequado durante a gestaçãos.

Tendo em vista que o peso ao nascer e as condições de saúde são determinadas por diversos fatores, é fundamental que se identifiquem quais são os que constituem risco para o nascimento de crianças com baixo peso ao nascer. A alimentação da gestante tem sido pouco analisada no Brasil. Dessa forma, este estudo tem como objetivo conhecer o estado nutricional da gestante adolescente, avaliado por meio de indicadores antropométricos e dietéticos, e avaliar o seu impacto no peso do recém-nascido.

\section{Métodos}

O estudo se constituiu na análise de um coorte histórico com 97 gestantes adolescentes e seus recémnascidos, atendidos no período de maio a junho de 2004. Foi realizado no Hospital e Maternidade Victor Ferreira do Amaral, hospital público, vinculado ao Sistema Único de Saúde (SUS), localizado em Curitiba, Paraná, que atende gestantes de médio e baixo risco.

As gestantes foram admitidas em trabalho de parto e avaliadas pela nutricionista responsável pelo Serviço de Nutrição Clínica, seguindo os protocolos de atendimento estabelecidos na instituição, incluindo avaliação do estado nutricional por meio de parâmetros antropométricos e dietéticos.

Foram incluídas as gestantes adolescentes, com faixa etária menor ou igual a 19 anos, de todas as raças, internadas em trabalho de parto, que consentiram a realização da avaliação nutricional, e os seus recémnascidos vivos. Foram excluídas as gestantes com gravidez múltipla, complicações gestacionais, idade gestacional menor do que 37 , que não apresentaram dados completos no prontuário ou não consentiram realizar a avaliação nutricional.

A coleta de dados maternos ocorreu no puerpério imediato (até 48 horas pós-parto) e incluiu peso préparto, idade, número de consultas pré-natais, estado civil, tipo de parto, hábito de fumar, número de gestações, peso e altura pré-gestacional, ganho de peso 
gestacional e consumo alimentar habitual referente ao final do terceiro trimestre. As medidas de peso e altura pré-gestacional foram obtidas pelas informações contidas na carteira do pré-natal. O peso pré-parto foi coletado do prontuário da paciente. Todas as informações foram confirmadas pela adolescente durante a entrevista, realizada por uma única pessoa, treinada e capacitada para esta função.

As gestantes adolescentes foram classificadas em primíparas ou multíparas, parto vaginal ou cesária, fumantes ou não-fumantes durante a gestação, se apresentavam idade ginecológica superior ou inferior a dois anos e se compareceram em menos de seis ou mais de seis consultas durante o pré-natal.

O estado nutricional materno foi avaliado mediante parâmetros antropométricos e dietéticos. A altura materna foi classificada em igual ou inferior a $1,50 \mathrm{~m}$ e maior que $1,50 \mathrm{~m}$. O IMC pré-gestacional foi calculado a partir do peso em quilogramas, dividido pelo quadrado da altura em metros $\left(\mathrm{kg} / \mathrm{m}^{2}\right)$, referido pela adolescente. As gestantes adolescentes foram classificadas em baixo peso (IMC $<19,8 \mathrm{~kg} / \mathrm{m}^{2}$ ), peso adequado/eutrofia (IMC entre 19,8 e $26 \mathrm{~kg} / \mathrm{m}^{2}$ ), sobrepeso (IMC entre 26 e $29 \mathrm{~kg} / \mathrm{m}^{2}$ ) e obesidade (IMC $>29 \mathrm{~kg} / \mathrm{m}^{2}$ ), conforme proposto pelo Institute of Medicine (IOM) ${ }^{11}$.

O ganho de peso gestacional (GPG) foi obtido por meio da subtração do peso pré-parto do peso pré-gestacional e avaliado pelo IMC pré-gestacional, proposto pelo $\mathrm{IOM}^{11}$. Adolescentes com baixo peso pré-gestacional devem ganhar até o final da gestação entre 12,5 e $18 \mathrm{~kg}$; as com peso adequado, entre 11,5 e $16 \mathrm{~kg}$; as com sobrepeso, entre 7 a $11,5 \mathrm{~kg}$; e as obesas devem ganhar em torno de $7 \mathrm{~kg}^{11}$. Dessa forma, o GPG foi classificado como insuficiente, adequado ou excessivo. O GPG também foi avaliado em quilogramas. As adolescentes foram classificadas em seis categorias: ganho de peso menor do que $6 \mathrm{~kg}$ e com intervalos de $3 \mathrm{~kg}$ entre cada categoria.

A necessidade calórica individual foi obtida por meio da soma do gasto energético basal, estimado segundo a idade materna ${ }^{12}$, acrescido de fator atividade ${ }^{12}$ e adicional energético de 300 calorias, recomendado para o período gestacional ${ }^{13}$. A necessidade protéica foi estabelecida segundo as recomendações do National Research Council ${ }^{13}$.

$\mathrm{O}$ instrumento utilizado para avaliar o consumo alimentar foi o inquérito de consumo habitual, referente ao final do terceiro trimestre da gestação. Os alimentos consumidos foram obtidos em medidas caseiras e a transformação em gramas e a análise do consumo de energia e proteína foram realizadas com utilização do software de avaliação nutricional DietWin Versão profissional $2.0^{14}$, cujos dados são compilações de várias tabelas de composição de alimentos nacionais e estrangeiros.

Os valores de calorias e proteínas encontrados por meio da análise do consumo alimentar foram comparados com as necessidades individuais estimadas. A definição de normalidade seguiu os critérios da Organização Mundial de Saúde (OMS), que estabelece o consumo em relação às necessidades diárias menor que $90 \%$ como insuficiente, entre 90 e $110 \%$ como adequado e maior que $110 \%$ como excessivo $^{15}$.

Para a avaliação do RN, foi coletado o peso ao nascer (PN) da carteira do RN. Foram considerados de baixo peso os RNs com peso inferior a $2.500 \mathrm{~g}$, com peso insuficiente os RNs com peso entre 2.500 e $2.999 \mathrm{~g}$, e peso favorável aqueles com mais de $3.000 \mathrm{~g}^{16}$.

Os dados foram analisados em distribuição contínua ou discreta. A análise descritiva dos dados incluiu a média, o desvio padrão e a variação. A associação entre as variáveis maternas (altura, IMC pré-gestacional, GPG e consumo) e o peso do $\mathrm{RN}$ foi analisada pelo teste de correlação de Spearman. Foi utilizado o pacote estatístico LHStat. O nível de significância de erro $\alpha$ foi de $5 \%(\mathrm{p}<0,05)$. A significância está apresentada na Tabela 1 e nas Figuras 1 e 2.

O estudo foi aprovado pelo Comitê de Ética em Pesquisa do Setor de Ciências da Saúde da Universidade Federal do Paraná (UFPR) em 7 de julho de 2004.

\section{Resultados}

A média de idade foi de 17,8 $\pm 1,12$ anos, com mínimo de 15 anos. A maioria das adolescentes (83) tinha idade igual ou superior a 17 anos.

Quanto ao estado civil, observou-se que a maioria (59) tinha união estável, 20 eram solteiras, 17 casadas e uma era viúva. Observou-se que 22 gestantes fumavam e 75 não fumavam durante a gestação.

O número de gestações por adolescente variou de um a três, sendo a maioria primíparas (73). Observou-se que 79 tiveram parto vaginal e 18 parto cesária. Nenhuma das adolescentes apresentou idade ginecológica inferior a dois anos. Identificou-se que 72 adolescentes compareceram a mais de seis consultas durante o prénatal e 25 a menos de seis consultas.

Observou-se que 11 gestantes apresentaram altura igual ou inferior a $1,50 \mathrm{~m}$, sendo que a maioria (86) apresentava altura superior a $1,50 \mathrm{~m}$ (Tabela 1). O IMC no início da gestação variou de 16,4 a $27,8 \mathrm{~kg} / \mathrm{m}^{2}$, com média de $21,1 \mathrm{~kg} / \mathrm{m}^{2}$. A maioria (64) iniciou a gestação com peso adequado (eutróficas), 28 apresentaram baixo peso e cinco foram classificadas como apresentando sobrepeso (Tabela 1). 
A média de GPG foi de $12,8 \pm 5,2 \mathrm{~kg}$ com variação de $2 \mathrm{~kg}$ de perda e um máximo de $30 \mathrm{~kg}$ de ganho. A maioria (60) apresentou ganho de peso inadequado e a adequação do GPG foi identificada em 37 gestantes (Tabela 1).

O relatório do consumo alimentar habitual no último trimestre mostrou o consumo calórico das gestantes adolescentes. $\mathrm{O}$ consumo energético médio foi de $2.567 \pm 921 \mathrm{kcal}$, com variação entre o mínimo de $1.016 \mathrm{kcal}$ e o máximo de $5.918 \mathrm{kcal}$. O total de calorias consumidas foi comparado com as necessidades individuais estimadas, que variaram de 2.141,46 a $2.783,32 \mathrm{kcal}$. A maioria das adolescentes apresentou consumo energético inadequado (Tabela 1).

A média de consumo protéico foi de 79,5 $\pm 2,85 \mathrm{~g} / \mathrm{dia}$. O consumo variou de $26,9 \mathrm{~g} / \mathrm{dia}$ a $152,5 \mathrm{~g} /$ dia. A maioria das adolescentes (63) apresentou consumo excessivo (Tabela 1).

A média de PN encontrada foi de 3.105 $\pm 391,8 \mathrm{~g}$. Entre os RNs, sete apresentaram baixo peso e 36 peso insuficiente, como mostra a Tabela 1 .

A altura materna e o peso ao nascer, como variáveis contínuas, apresentaram correlação positiva e significante $\left(r_{s}=0,19 ; p=0,01 ;\right.$ Figura 1$)$. Os RNs de adolescentes com altura igual ou inferior a $1,50 \mathrm{~m}$ pesavam, em média, $295 \mathrm{~g}$ a menos do que os nascidos de adolescentes mais altas (Tabela 1).

Recém-nascidos de adolescentes que iniciaram a gestação com baixo peso apresentaram a maior média de PN, enquanto que os RNs de adolescentes com sobrepeso apresentaram a menor média (Tabela 1). Observou-se uma correlação inversa e estatisticamente

Tabela 1 - Interação entre estado nutricional materno e peso ao nascer (PN).

\begin{tabular}{|c|c|c|c|c|c|c|c|c|c|}
\hline Variáveis & $\mathbf{N}$ & $\%$ & $\begin{array}{l}\text { Média de PN } \\
\text { (gramas) }\end{array}$ & $\begin{array}{l}\text { RN baixo } \\
\text { peso (n) }\end{array}$ & $\%$ & $\begin{array}{c}\text { RN peso } \\
\text { insuficiente (n) }\end{array}$ & $\%$ & $\begin{array}{c}\text { RN peso } \\
\text { favorável }(\mathrm{n})\end{array}$ & $\%$ \\
\hline \multicolumn{10}{|l|}{ (A) Alfura maferna } \\
\hline$\leq 1,50 \mathrm{~m}$ & 11 & 11,3 & 2.844 & 3 & 27,3 & 4 & 36,4 & 4 & 36,4 \\
\hline$>1,50 \mathrm{~m}$ & 86 & 88,7 & $3.138,8$ & 4 & 4,7 & 32 & 37,2 & 50 & 58,1 \\
\hline \multicolumn{10}{|l|}{$\begin{array}{l}\text { (B) IMC pré- } \\
\text { gestacional }\end{array}$} \\
\hline Baixo peso & 28 & 28,9 & 3.125 & 2 & 7,1 & 12 & 42,9 & 14 & 50 \\
\hline Eutrofia & 64 & 66,0 & 3.102 & 4 & 6,3 & 23 & 35,9 & 37 & 57,8 \\
\hline Sobrepeso & 5 & 5,2 & 3.035 & 1 & 20 & 1 & 20 & 3 & 60 \\
\hline \multicolumn{10}{|l|}{$\begin{array}{l}\text { (C) Classes de GPG } \\
(\mathrm{kg})\end{array}$} \\
\hline$<6$ & 12 & 12,4 & 2.902 & 2 & 16,7 & 5 & 41,7 & 5 & 41,7 \\
\hline 6,1 a 9 & 10 & 10,3 & 2.892 & 2 & 20 & 3 & 30 & 5 & 50 \\
\hline 9,1 a 12 & 24 & 24,7 & 3.013 & 1 & 4,2 & 15 & 62,5 & 8 & 33,3 \\
\hline 12,1 a 15 & 27 & 27,8 & 3.166 & 2 & 7,4 & 7 & 25,9 & 18 & 66,7 \\
\hline 15,1 a 18 & 11 & 11,3 & 3.234 & 0 & 0 & 3 & 27,3 & 8 & 72,7 \\
\hline$>18,1$ & 13 & 13,4 & 3.392 & 0 & 0 & 3 & 23,1 & 10 & 76,9 \\
\hline \multicolumn{10}{|l|}{$\begin{array}{l}\text { (D) Classes de GPG } \\
\text { (IOM) }\end{array}$} \\
\hline GPG insuficiente & 39 & 40,2 & 2.957 & 5 & 12,8 & 18 & 46,2 & 16 & 41,0 \\
\hline GPG adequado & 37 & 38,1 & 3.124 & 1 & 2,7 & 15 & 40,5 & 21 & 56,8 \\
\hline GPG excessivo & 21 & 21,6 & 3.348 & 1 & 4,8 & 3 & 14,3 & 17 & 81,0 \\
\hline \multicolumn{10}{|l|}{ (E) Consumo calórico } \\
\hline Menor que $90 \%$ & 39 & 40,2 & 3.109 & 4 & 10,3 & 12 & 30,8 & 23 & 59,0 \\
\hline De 90 a $110 \%$ & 14 & 14,4 & 3.196 & 1 & 7,1 & 4 & 28,6 & 9 & 64,3 \\
\hline Maior que $110 \%$ & 44 & 45,4 & 3.074 & 2 & 4,5 & 20 & 45,5 & 22 & 50,0 \\
\hline \multicolumn{10}{|l|}{ (F) Consumo protéico } \\
\hline Menor que $90 \%$ & 17 & 17,5 & 3.219 & 2 & 11,8 & 3 & 17,6 & 12 & 70,6 \\
\hline De 90 a $110 \%$ & 17 & 17,5 & 2.998 & 2 & 11,8 & 8 & 47,1 & 7 & 41,2 \\
\hline Maior que $110 \%$ & 63 & 64,9 & 3.104 & 3 & 4,8 & 25 & 39,7 & 35 & 55,6 \\
\hline
\end{tabular}

(A) Altura pré-gestacional (metro) versus PN (gramas) $r_{s}=0,19 * ;(B) I M C$ pré-gestacional $\left(\mathrm{kg} / \mathrm{m}^{2}\right)$ versus $\mathrm{PN}$ (gramas) $\mathrm{r}_{\mathrm{s}}=0,01$; IMC pré-gestacional (classes) versus PN (gramas) $r=-0,28 * *$; IMC pré-gestacional (classes) versus PN (classes) $r=-0,23 *$; (C) GPG (classes kg) versus PN (classes) $r=0,26 * *$; $G P G$ (classes $\mathrm{kg}$ ) versus PN (gramas) $r_{s}=0,36 * *$; (D) GPG (classes IOM) versus PN (gramas) $r_{s}=0,24^{* *}$; (E) Consumo calórico (kcal) versus PN (gramas) $r_{s}=-0,07$; (F) Consumo protéico (g) versus $P N$ (gramas) $r_{s}=-0,07$; Consumo protéico (classes) versus $P N$ (gramas) $r_{s}=-0,24$ * *

$\mathrm{RN}=$ recém-nascido; IMC=índice de massa corporal; $\mathrm{GPG}=$ ganho de peso gestacional; $I O M=$ Institute of Medicine; $r_{s}=$ coeficiente de correlação de Spearman; ${ }^{*} p<0,05 ;{ }^{*} p<0,01$ 
significante entre o peso ao nascer, tanto como variável contínua (gramas) quanto em classes, de baixo peso a favorável, com o IMC pré-gestacional estratificado em classes de baixo peso a sobrepeso $\left(r_{s}=0,28 ; p=0,002\right.$ e $r=-0,23 ; p=0,01$, respectivamente). Entretanto, não foi observada correlação entre as variáveis quando elas foram avaliadas de forma contínua.

A correlação entre GPG e PN foi analisada de várias formas. Inicialmente verificou-se a correlação entre GPG e PN como variáveis contínuas (Figura 2). Observou-se uma correlação positiva e significante $\left(r_{s}=0,41, p=0,0000\right)$. A associação de classes de GPG em $\mathrm{kg}$ com as classes de PN e o PN como variável contínua também evidenciou correlação positiva e significante $\left(r_{s}=0,26, p=0,004 ; r_{s}=0,36, p=0,0001\right)$ conforme Tabela 1.

Posteriormente, as variáveis foram analisadas alocando a classes de GPG, de insuficiente a excessivo, e o PN de forma contínua (gramas). Nesta situação, as correlações foram positivas e significantes $\left(r_{s}=0,24\right.$; $\mathrm{p}=0,006)$, como mostra a Tabela 1 . Os RNs de ado-

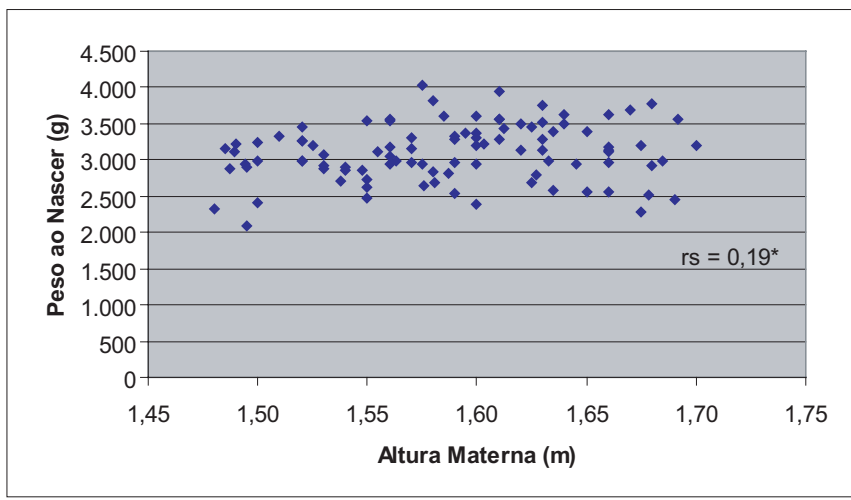

Altura materna (metro: variável contínua) versus peso ao nascer (gramas: variável contínua) $r s=0,19 ; p<0,05$.

$\mathrm{rs}=$ coeficiente de correlação de Spearman

Figura 1 - Interação entre altura materna $(\mathrm{m})$ e peso ao nascer (g).

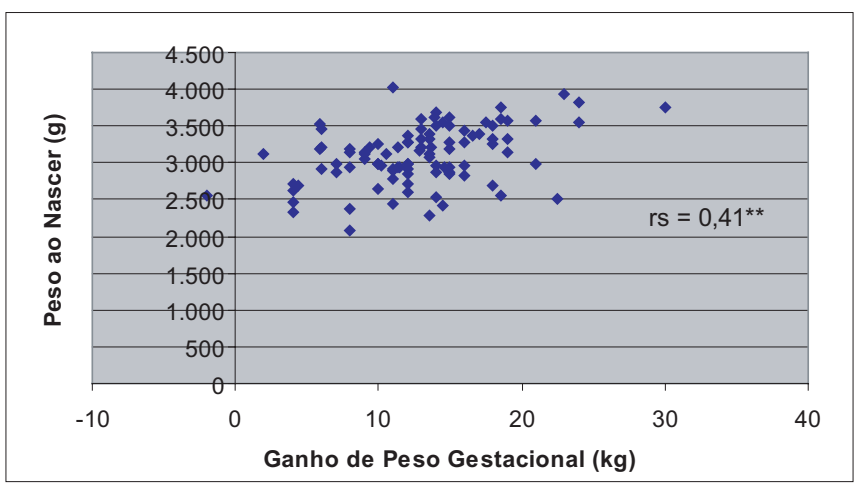

Ganho de peso gestacional (kg: variável contínua) versus peso ao nascer (gramas: variável contínua) $r_{s}=0,41 ; p<0,01$. $r=$ coeficiente de correlação de Spearman.

Figura 2 - Interação entre ganho de peso gestacional $(\mathrm{kg})$ e peso ao nascer (g) lescentes com GPG insuficiente apresentaram a menor média de PN quando comparados aos RNs de adolescentes que apresentaram ganho de peso adequado ou excessivo (Tabela 1).

Não foi observada correlação entre consumo calórico no terceiro trimestre e PN ( $\left.\mathrm{r}_{\mathrm{s}}=0,07 ; \mathrm{p}=0,22\right)$. Recémnascidos de adolescentes com consumo calórico excessivo tiveram a menor prevalência de baixo peso ao nascer $\mathrm{e}$ os RNs de adolescentes com adequado consumo calórico tiveram a maior média de PN (Tabela 1).

Observou-se uma correlação inversa estatisticamente significante entre consumo protéico no terceiro trimestre, estratificado em classes de insuficiente a excessivo, com o peso ao nascer como variável contínua e estratificado ( $r s=-0,24, p=0,007$ e rs $=-0,25$; $\mathrm{p}=0,005$, respectivamente). Não foi observada correlação entre as variáveis quando elas foram avaliadas de forma contínua.

\section{Discussão}

O peso ao nascer é o fator isolado mais importante na determinação da sobrevivência infantil. Crianças com peso menor do que 2.500 gramas apresentam maiores riscos de adoecer ou morrer no primeiro ano de vida ${ }^{2}$.

$\mathrm{O}$ baixo peso tem sido encontrado com maior freqüência em RNs de adolescentes ${ }^{1,17,18}$. Neste estudo, $7 \%$ dos RNs apresentaram baixo peso e $37 \%$ peso insuficiente.

Recém-nascidos de mães adolescentes apresentam um risco duas vezes maior de baixo peso e três vezes maior de mortalidade neonatal em relação aos nascidos de mães adultas ${ }^{15}$. Na Tanzânia, o risco de baixo peso ao nascer é quatro vezes maior para gestantes adolescentes quando comparado com gestantes adultas ${ }^{19}$.

Em um estudo realizado no Município do Rio de Janeiro entre 1996 a 1998, foi constatado maior concentração de recém-nascidos com baixo peso ao nascer em filhos de mulheres entre 15 e 19 anos, quando comparadas com mulheres com 20 a 24 anos $^{1}$. Resultados semelhantes foram encontrados no município de Rio Branco, no estado do Acre, onde a freqüência de baixo peso ao nascer foi maior em mães adolescentes $(14,9 \%)$ quando comparadas com mães adultas $(11 \%)^{18}$.

Alguns indicadores do estado nutricional materno têm sido associados com o peso ao nascer. A altura materna, além de exercer impacto sobre o peso ao nascer, tem sido utilizada para avaliar os riscos de mortalidade perinatal, neonatal e infantil e a performance lactacional ${ }^{3}$. Essa relação é observada entre gestantes adultas e adolescentes ${ }^{11}$. No Brasil, o ponto de corte 
mais utilizado para a estatura materna é de $150 \mathrm{~cm}$, abaixo do qual observam-se maiores riscos ${ }^{20}$.

Concordantes aos achados deste estudo diversos autores têm encontrado relação positiva entre altura materna e peso ao nascer ${ }^{2,8,19,20}$. A altura materna pode representar o principal determinante para o baixo peso ao nascer. Recém-nascidos de gestantes com altura inferior a $150 \mathrm{~cm}$ apresentaram, em média, $287 \mathrm{~g}$ a menos quando comparadas com os RNs de gestantes com altura superior ${ }^{20}$. Cada $10 \mathrm{~cm}$ adicionados à altura materna corresponderam a um aumento de $172 \mathrm{~g}$ no peso do $\mathrm{RN}^{9}$.

No presente estudo, foi encontrada correlação inversa e estatisticamente significante entre o IMC pré-gestacional e o peso ao nascer. Há controvérsias quanto à influência do IMC pré-gestacional no peso ao nascer. Alguns autores têm encontrado relação positiva ${ }^{2}$. Colletto e Segre ${ }^{21}$ verificaram associação positiva para gestantes com filho único, mas não para os gêmeos. Outros estudos não têm verificado associação significante entre IMC pré-gestacional e peso do recém-nascido ${ }^{7,20,22}$. Porém, tem sido observada associação estatisticamente significante entre a desnutrição no final do período gravídico e baixo peso ao nascer ${ }^{7,22}$.

Embora se tenha observado uma correlação inversa entre IMC pré-gestacional e peso ao nascer, é indiscutível a importância do estado nutricional antes e durante a gestação na evolução do concepto. É necessária a análise conjunta do IMC pré-gestacional e do ganho de peso na avaliação do estado nutricional de gestantes ${ }^{3}$.

Observou-se, neste estudo, que aquelas que iniciaram a gestação com baixo peso apresentaram a maior média de peso ao nascer, maior adequação quanto ao ganho de peso segundo as recomendações propostas pelo IOM e maior média de ganho de peso gestacional. Adolescentes que iniciam a gestação com baixo peso devem ganhar mais peso quando comparadas com as eutróficas e com sobrepeso ${ }^{11}$. O peso pré-gestacional inadequado acompanhado por ganho de peso insuficiente aumenta o risco de baixo peso ao nascer e a mortalidade perinatal, neonatal e infantil ${ }^{3}$.

A relação entre ganho de peso gestacional e peso ao nascer foi avaliada de várias formas e, em todas as situações, foi verificada correlação positiva e significante. Observou-se que o peso do $\mathrm{RN}$ pode aumentar com a adequação do ganho de peso durante a gestação.

Está bem demonstrada na literatura a relação positiva entre ganho de peso gestacional e peso ao nascer ${ }^{2,8,19,20}$. Gestantes que apresentam ganhos de peso inferiores ou iguais a $10 \mathrm{~kg}$ têm maior risco de terem recém-nascidos de baixo peso e aquelas com ganhos inferiores a $12 \mathrm{~kg}$ têm maior risco de terem RNs com ganho de peso insuficiente ${ }^{23,24}$. Entretanto, o ganho de peso excessivo, acima de $16 \mathrm{~kg}$, não representa incremento adicional no peso ao nascer, simplesmente incorpora-se ao organismo materno ${ }^{20}$. Entretanto, é importante ressaltar que o ganho de peso excessivo tem sido associado com gestações de alto risco, incluindo, além de complicações do parto, anemia materna, parto pré-termo, macrossomia e mortalidade infantil ${ }^{24}$.

O ganho de peso materno, diferente da altura e do peso pré-gestacional, pode ser controlado durante a assistência pré-natal, possibilitando a recuperação das gestantes desnutridas e a diminuição considerável do risco de nascimento de crianças com baixo peso ${ }^{25}$.

No presente estudo, não foi encontrada correlação significativa entre o consumo calórico no terceiro trimestre de gestação e o peso ao nascer. Recémnascidos de adolescentes com adequado consumo calórico tiveram a maior média de PN. A influência do consumo calórico no peso ao nascer é controversa. Alguns estudos encontraram relação positiva entre as variáveis ${ }^{23}$. Entretanto, outros não têm verificado a mesma relação $0^{9,26}$.

$\mathrm{Na}$ ausência de deficiência de nutrientes específicos, o efeito da dieta materna sobre o crescimento fetal pode ocorrer somente quando a mulher tiver baixo IMC pré-gestacional e sua ingestão energética não atingir as necessidades recomendadas durante a gestação ${ }^{27}$.

As controvérsias permanecem em relação ao consumo protéico. A relação positiva tem sido observada em diferentes fases da gestação, como em até 16 semanas gestacionais ${ }^{26}$. O consumo de proteínas além do recomendado não tem efeitos positivos sobre o peso ao nascer. A média de consumo maior ou igual a $85 \mathrm{~g} /$ dia associou-se com a diminuição de $71 \mathrm{~g}$ no peso ao nascer. Já a ingestão menor do que 50 g/dia levou a uma redução de $77 \mathrm{~g}$ no peso ao nascer ${ }^{28}$. Entretanto, associação entre o declínio do peso ao nascer e o alto consumo protéico não foi confirmado por outros autores $^{26}$. Neste estudo, foi observada uma correlação inversa estatisticamente significante entre consumo protéico no terceiro trimestre e o peso ao nascer. As gestantes com consumo protéico insuficiente tiveram maior média de ganho de peso e maior média de peso ao nascer quando comparadas com aquelas com consumo adequado ou excessivo.

A relação entre consumo de nutrientes durante a gestação e peso ao nascer pode ser indireta e modulada pelo ganho de peso gestacional. Além disso, os efeitos nutricionais no feto variam conforme o estágio da gestação, pois o desenvolvimento fetal e a necessidade de nutrientes são diferentes no decorrer da gestação. Dessa forma, a percepção da influência do consumo 
alimentar no peso ao nascer dependerá do momento em que o consumo foi avaliado ${ }^{26}$.

A adolescente que não tem uma alimentação nutricionalmente adequada ou apresenta baixo peso pré-gestacional certamente inicia a gestação com as reservas limitadas ou ausentes, o que aumenta o risco de baixo peso ao nascer e prematuridade ${ }^{29}$. A intervenção nutricional precoce irá exercer uma influência positiva sobre este prognóstico ${ }^{29}$.

Com base nos resultados obtidos, conclui-se que o estado nutricional pregresso, avaliado pela altura prégestacional, e o estado nutricional imediato, avaliado pelo ganho de peso gestacional, influenciam o peso do RN de gestantes adolescentes, demonstrando o impacto do estado nutricional materno nas condições de nascimento.

Apesar de o IMC pré-gestacional ter apresentado correlação inversa com o peso ao nascer, sua determinação no início da gestação é fundamental para determinar qual será o ganho de peso adequado. Aquelas que iniciam a gestação com baixo IMC, desde que ganhem o peso proposto pelo IOM, podem ter filhos com adequado peso ao nascer. Este é um achado importante, visto que é mais fácil controlarmos o ganho de peso com a assistência pré-natal adequada, envolvendo equipe multidisciplinar, incluindo nutricionistas, do que modificarmos o IMC das adolescentes que se encontram em idade fértil.

Neste estudo, não foi demonstrada associação significativa entre o consumo calórico com o peso ao nascer, entretanto, observou-se que a maioria das adolescentes avaliadas não está consumindo uma dieta adequada, ressaltando a importância de um acompanhamento nutricional efetivo, que oriente na seleção de alimentos compatíveis com o seu estado fisiológico e que corrija as inadequações de ganho de peso detectadas.

\section{Agradecimentos}

Aos Professores Doutores Raul von der Heyde (UFPR) e Claudio Loesch (FURB) pelo auxílio na análise estatística dos dados.

\section{Referências}

1. Gama SGN, Szwarcwald CL, Leal MC, Theme Filha MM. Gravidez na adolescência como fator de risco para o baixo peso ao nascer no Município do Rio de Janeiro, 1996 a 1998. Rev Saúde Pública. 2001;35(1):74-80.

2. Rocha DS, Pereira Netto M, Priore SE, Lima NMM, Rosado LEFPL, Franceschini SCC. Estado nutricional e anemia ferropriva em gestantes: relação com o peso da criança ao nascer. Rev Nutr. 2005; 18(4):481-9.

3. Pan American Health Organization. Maternal nutrition and pregnancy outcomes: anthropometric assessment. Washington, D.C: PAHO; 1991. (Scientific Publication, 529).

4. Ramakrishnan U. Nutrition and low birth weight: from research to practice. Am J Clin Nutr. 2004;79(1):17-21. Review.

5. Eisenstein E, Coelho KSC, Coelho SC, Coelho MASC. Nutrição na adolescência. J Pediatr (Rio de J). 2000; 76 Supl 3:S263-S274.

6. Costa MCO, O Neto AF. Abordagem nutricional de gestantes e nutrizes adolescentes: estratégia básica na prevenção de riscos. J Pediatr (Rio de J). 1999;75(3): 161-6.

7. Furlan JP, Guazzelli CAF, Papa ACS, Quintino MP, Soares RVP, Mattar R. A influência do estado nutricional da adolescente grávida sobre o tipo de parto e o peso do recém-nascido. Rev Bras Ginecol Obstet. 2003;25(9):625-30.

8. Lima GSP, Sampaio HAC. Influência de fatores obstétricos, socioeconômicos e nutricionais da gestante sobre o peso do recém-nascido: estudo realizado em uma maternidade de Teresina, Piauí. Rev Bras Saúde Matern Infant. 2004;4(3):253-61.

9. Mathews F, Yudkin P, Neil A. Influence of maternal nutrition on outcome of pregnancy: prospective cohort study. BM. 1999;319(7206):339-43.
10. Juzwiak CR, Lopez FA. Programação metabólica: a influência da nutrição intra-útero na saúde da vida adulta. Nutr Saúde Perform. 2006;30:22-7.

11. Institute of Medicine. National Academy of Sciences. Nutrition during pregnancy and lactation. An implementation guide. Washington, D.C.: National Academy Press; 1992.

12. Food and Agriculture Organization (FAO). World Health Organization (WHO). United Nations. Energy and protein requirements. Geneve: WHO; 1985. (Technical Report Series, 724).

13. National Research Council. Recommended dietary allowances. $10^{\text {th }}$ ed. Washington, D.C.: National Academy Press; 1989.

14. Dietwin. Software de avaliação nutricional. Versão profissional 2.0. Porto Alegre: Brubins; 2004.

15. World Health Organization. Diet, nutrition and the prevention of chronic diseases. Geneva: WHO; 1990.

16. Puffer RR, Serrano CV. Patterns of birthweights. Washington, D.C.: Pan American Health Organization; 1987. (Scientific Publication, 504).

17. Roth J, Hendrickson J, Schilling M, Stowell DW. The risk of teen mothers having low birth weight babies: implications of recent medical research for school health personnel. J Sch Health. $1998 ; 68(7): 271-5$.

18. Aquino-Cunha M, Queiroz-Andrade M, Tavares-Neto J, Andrade T. Gestação na adolescência: relação com o baixo peso ao nascer. Rev Bras Ginecol Obstet. 2002; 24(8):513-9.

19. Shirima C, Kinabo JL. Nutritional status and birth outcomes of adolescent pregnant girls in Morogoro, Coast, and Dar es Salaam regions, Tanzania. Nutrition. 2005;21(1):32-8. 
20. Franceschini SCC, Priore SE, Pequeno NPF, Silva DG, Sigulem DM. Fatores de risco para o baixo peso ao nascer em gestantes de baixa renda. Rev Nutr. 2003;16(2):171-9.

21. Colletto GM, Segre CA. Lack of effect of maternal body mass index on anthropometric characteristics of newborns in twin gestations. Genet Mol Res. 2005;4(1):47-54.

22. Sinisterra Rodriguez $O$, Szarfarc SC, Benicio MHA. Anemia e desnutrição maternas e sua relação com o peso ao nascer. Rev Saúde Pública. 1991;25(3):193-7.

23. Lizo CLP, Lopez FA, Segre CAM. Influência do estado nutricional, ganho de peso gestacional e tabagismo maternos sobre o peso e comprimento de recém-nascidos normais em população de alta condição sócio-econômica. Pediatr Mod. 1994;30(2):287-304.

24. Howie LD, Parker JD, Schoendorf KC. Excessive maternal weight gain patterns in adolescents. J Am Diet Assoc. 2003;103(12): 1653-7.
25. Brasil ALD, Demarchi ALG. Nutrição na gestação e na lactação. In: Lopez FA, Brasil ALD, editores. Nutrição e dietética em clínica pediátrica. São Paulo: Atheneu; 2003. p. 3-16.

26. Moore VM, Davies M, Willson KJ, Worsley A, Robinson JS. Dietary composition of pregnant women is related to size of the baby at birth. J Nutr. 2004;134(7):1820-6.

27. Rosso P. Nutrition and metabolism in pregnancy. New York: Oxford University Press; 1990.

28. Sloan NL, Lederman SA, Leighton J, Himes JH, Rush D. The effect of prenatal dietary protein intake on birth weight. Nutr Res. 2001;21(1):129-39.

29. Position of the American Dietetic Association: nutrition management of adolescent pregnancy. J Am Diet Assoc. 1989;89(1): 104 